\title{
Expect the unexpected
}

\author{
When you have discovered something unusual, trust your instinct and pursue it with determination and \\ enthusiasm, says Renren Deng.
}

M $y$ research interests are in the field of lanthanide-doped upconversion nanocrystals, which are inorganic nanophosphors able to convert a beam of near-infrared light into one with a shorter wavelength in the visible or even ultraviolet range. Because of their intriguing optical phenomena, these nanocrystals are promising for many applications such as biological imaging, data storage, security printing, or 3D displays. The first time I heard about upconversion nanocrystals was in 2009 when I was looking for an advisor for my $\mathrm{PhD}$ studies and first met with Xiaogang Liu, a professor in the Department of Chemistry at the National University of Singapore. Before that, I was at Zhejiang University in China for my undergraduate studies and my thesis project mainly focused on the development of chemical sensors based on fluorescent organic molecules. Despite having no background in nanoscience, I decided to join Xiaogang Liu's group as a graduate student and to start working on upconversion nanocrystal-based research because of his encouragement and his belief that I could do it well. More importantly, it was also because I trusted my instinct and was not afraid to face the challenges; this led to the publication of my work on page 237 of this issue.

In early 2010, just the second week after I went into the lab and started my experiments, I began working on the synthesis of upconversion nanocrystals. I was following a procedure reported in the literature and I expected that my nanocrystals dispersed in a solution would emit red light when excited with a 980-nm laser. However, to my surprise, I observed unexpectedly that when the nanocrystal solution was gently shaken in front of the laser beam, it emitted yellow rather than red light. By this time, I had already prepared many different batches of upconversion nanocrystals, but this was the first time that I had observed such a phenomenon. After discussing with my colleagues in the group who had more experience than me in this field, I realized that the observation could be important. Full of excitement, I kept working on the

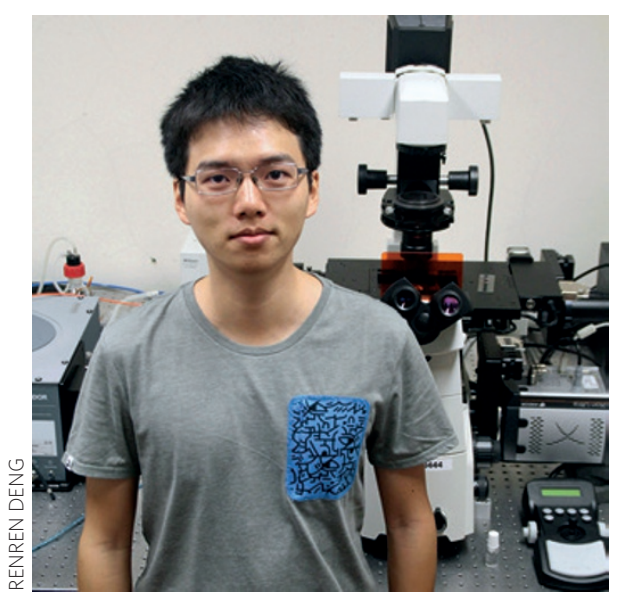

characterization of these nanocrystals. Unfortunately, this proved more challenging than I had anticipated; because I had to continuously shake the particle solution to keep the emission colour changed, I could not place it into a fixed sample chamber for characterization. I still remember the frustration of not being able to perform spectroscopic investigations while shaking the sample solution. So I decided to put this work aside for a while, hoping that one day I could find an explanation for this phenomenon as I continued to expand my knowledge in the field.

In 2012, I was involved in a project on enhanced upconversion through energy clustering, the results of which were published in Nature Materials $(13,157-162 ; 2014)$. During the course of the project, I was intrigued by the depth of complexity of the energy transfer mechanism that governs the kinetics of the upconversion process. For example, I learned that by varying the excitation condition of intermediate excited states of lanthanides it should have an impact on the colour of the light emission by the nanocrystals. Having gained this insight into the fundamental physical nature of upconversion emission, I wondered whether the colour change caused by shaking was due to a non-equilibrium multiphoton upconversion process, in which the emission rate of different excited states was dynamically changing, leading to the variation of emission intensity ratios of peaks with different wavelengths.

To trigger this non-equilibrium optical process, I first fabricated a rough homemade mechanical fan with paperboard blades as an optical chopper to produce a pulse stream from a continuous-wave laser beam. I observed a colour change when I used the chopper-modulated laser to excite a solution containing the same upconversion nanoparticles I had prepared previously for the shaking experiments. Importantly, I was also able to measure the emission spectra and validate my hypothesis that the colour change was caused by the variation in emission intensity ratios of different emission peaks.

The preliminary results of tuning the emission colour of the upconversion nanocrystals by pulsed operation of the laser were exciting for my advisor. With help from other group members and our collaborators, we then took a decisive step forward and prepared multicolouremissive nanocrystals adopting a sophisticated multilayer core-shell structure. We subsequently succeeded in fine-tuning emission in the full colour range by excitation-pulse modulation and in the application of these fascinating optical nanomaterials for volumetric 3D full-colour displays.

I noticed an unusual phenomenon that could not be explained in the early stage of my PhD. Luckily, I was not intimidated by the numerous obstacles at the outset and finally solved the puzzle just before my doctoral degree was granted in July 2014. Given the fact that much of scientific research is about making discoveries and searching for things that are unusual and unexpected, my experience has taught me that the willingness to pay attention to such things, trusting your instinct, and being patient may also be essential.

RENREN DENG is a postdoctoral researcher in the Department of Chemistry, National University of Singapore, Singapore 117543, Singapore.

e-mail: chmdr@nus.edu.sg 